\title{
SIW Textile Antennas as a Novel Technology for UWB RFID Tags
}

\author{
Sam Lemey, Hendrik Rogier \\ INTEC Department \\ iMinds/Ghent University \\ Ghent, Belgium \\ Sam.Lemey@intec.ugent.be
}

\begin{abstract}
A wearable ultra-wideband (UWB) textile antenna based on substrate integrated waveguide technology is presented for application in the next generation radio-frequency identification (RFID) systems operating in the low-duty cycle restricted [3.4-4.8] GHz UWB band. The design of a UWB antenna for application in smart textiles is very challenging. For end users, the antenna should be unobtrusively integrated into smart textile and comfortable to wear. From a system point of view, good impedance matching and radiation characteristics over a very large bandwidth are of major importance, even under harsh operating conditions. Our approach consists of applying copper tube eyelets to implement a cavity-backed slot antenna in textile materials. A prototype was realized and measured. The measured return loss characteristic and radiation performance after bending and deploying the antenna on different human body parts prove that our antenna is extremely suited for on-body use. A -10 dB-bandwidth of $1.33 \mathrm{GHz}$, or 33 $\%$, was realized, in which the radiation pattern remains quasi invariable.
\end{abstract}

Keywords - Radio-frequency identification (RFID), substrate integrated waveguide (SIW), textile antenna, ultra-wideband (UWB), wearable antenna.

\section{INTRODUCTION}

$\mathrm{R}$ apid developments in smart fabric interactive textile (SFIT) systems promise to revolutionize many aspects of a person's daily life. Potential applications are, amongst others, monitoring and localization of first responders during interventions and monitoring vital signs and position of patients in hospitals and home-care scenarios. For instance, [1] designed a wearable multiband active antenna for use in Global Positioning System (GPS) applications and demonstrated the possibility to track first responders in outdoor locations. Reference [2] showed that GPS users could expect positional accuracies within approximately $5 \mathrm{~m}$ in open sky settings. Moreover, solutions based solely on GPS are not optimal in indoor scenarios.

Recent developments in radio-frequency identification (RFID) systems paved the way to accurate submeter localization in indoor and confined areas [3], [4]. RFID systems consist of readers on the one hand, and tags applied to objects, on the other hand. The narrow band nature of first and second generation RFID systems, operating in the ultra-high-

Part of this work was supported by BELSPO through the IAP Phase VII BESTCOM project. frequency (UHF) band and using standard continuous wave communication, makes them sensitive to narrowband and multiuser interference, vulnerable to multipath signal cancellation, and unsuitable for accurate localization [5]. Therefore, next generation RFID systems adopt the impulse radio ultra-wideband (IR-UWB) implementation to transmit (sub)nanosecond duration pulses. This enables them to provide both reliable identification [5] and high-accuracy localization of objects [3],[4]. Besides real-time localization at submeter level, these next generation RFID systems feature extremely low power consumption [6], low cost and small size. Hence, they are very suitable for integration into SFIT textile systems, to enable high-definition localization of persons in indoor locations or confined areas. Implementing multiple of judiciously positioned RFID tags could even allow reconstructing the position and orientation of the human body to enable augmented sensing, such as fall detection for the elderly. The U.S. Federal Communications Commission (FCC) has allocated a [3.1-10.6] GHz-block of radio spectrum for unlicensed UWB transmission at a maximum power spectral density of $-41.3 \mathrm{dBm} / \mathrm{MHz}$ [7]. They defined an UWB signal as a signal with instantaneous spectral occupancy in excess of $500 \mathrm{MHz}$ or a fractional bandwidth of $20 \%$ or more. The European Commission has issued an EC decision which states that UWB devices can use the [3.4-4.8] GHz and the [6.0-8.5] $\mathrm{GHz}$ band with the same maximum power spectral density as in the U.S., provided that signals in the lower frequency band meet a low duty cycle restriction [8].

Obviously, the ultra-wide bandwidth implies substantial different antenna topologies and propagation aspects as compared to narrow band systems, which makes practical antenna design more challenging [9]. Besides the fact that matching and efficient radiation should be ensured over an ultra-wide bandwidth, UWB antenna radiation patterns should be as constant as possible over the entire bandwidth to minimize direction-specific distortion of UWB waveforms [9]. Moreover, a wearable on-body antenna should exhibit robust impedance matching and radiation performance, independent of the wearer's morphology and of the antenna's position on the body. This can be achieved by designing the antenna in such a way that it radiates away from the body. Since power is radiated in the desired directions instead of being absorbed by the user's body, such a design strategy also results in a 
safer and more power-efficient design. Finally, end users of SFIT systems impose stringent requirements in terms of wearability and comfort. Hence, each component should be low-profile, flexible and breathable, making textile antennas very suitable for unobtrusive integration in SFIT systems.

In this contribution, we present a robust wearable UWB textile cavity-backed slot antenna in substrate integrated waveguide (SIW) technology, for operation in the low duty cycle restricted [3.4-4.8] GHz band. More specifically, we design for a maximum -10dB impedance bandwidth within this band, in the meantime maintaining robust and efficient operation, since [10] demonstrates a ranging error of only 1.63 $\mathrm{cm}$ when using an IR-UWB pulse with a $500 \mathrm{MHz}$ bandwidth at a center frequency of $4.25 \mathrm{GHz}$ and [11] stated that a larger bandwidth is beneficial for reducing the ranging error. Cavitybacked slot antennas have the potential to exhibit stable characteristics in the vicinity of the human body, since they only transmit/receive power in one hemisphere and reduce radiation leakage in the opposite hemisphere. By implementing the cavity with SIW technology, a low-profile single substrate antenna can be realized. Different textile SIW cavity-backed antennas were already realized by using conductive yarn [12] or tubular eyelets [13]. Yet, on the one hand, both designs are narrowband, as they rely on a single resonant frequency excitation. On the other hand, [14],[15] describe techniques to increase the impedance bandwidth. The latter technique is effectively exploited in [16] to realize a wideband textile SIW cavity-backed antenna with an impedance bandwidth of $409 \mathrm{MHz}$ or $15.1 \%$, thereby covering the $2.45 \mathrm{GHz}$ Industrial, Scientific and Medical band as well as both up- and downlink of the 4G LTE band 7. [16] also demonstrates the potential to integrate electronic circuits below the antenna cavity and solar cells on top of the cavity beside the antenna slot. The antenna topology, its construction process and the measures taken to achieve a robust wearable UWB textile antenna are discussed in Section II. The performance of the antenna in free space and when deployed on different parts of the human body is evaluated and documented in Section III.

\section{ANTENNA DESIGN AND TOPOLOGY}

\section{A. Antenna design considerations}

The most widely accepted definition of an UWB signal, formulated by the FCC, requires that the antenna exhibits a $-10 \mathrm{~dB}$ bandwidth in excess of $500 \mathrm{MHz}$ and/or a fractional bandwidth higher than $20 \%$. Our main design goal is therefore to design a wearable textile antenna with UWB behavior in the low duty cycle restricted [3.4-4.8] GHz band. The deployment of a wearable antenna on the human body in conjunction with several detrimental conditions, such as crumpling, bending and moisture regain will cause a shift in bandwidth and resonant frequency [17] which can be minimized by a suitable choice of antenna topology, antenna materials and implementation technology. Obviously, in all realistic deployment conditions, the antenna topology should also maintain its UWB behavior. These choices will be discussed in Section II.B and Section II.C. Once a suitable topology is chosen, the antenna will be designed for maximum bandwidth, in combination with large radiation efficiency. Moreover, we aim for a stable and broad radiation pattern over the entire bandwidth, to minimize direction-specific distortion of the UWB waveforms, in the meantime maintaining a compact and low-profile structure.

\section{B. Antenna design}

The configuration of our proposed textile UWB SIW cavity-backed slot antenna is shown in Fig. 1. A cavity-backed slot antenna is selected because such a topology demonstrates an excellent isolation from its environment [14], making it not only extremely suitable for on-body deployment, but also for the integration of additional electronic hardware [16]. By deploying the antenna on the human body in such a way that the slot is oriented away from the body, the antenna mainly radiates in that hemisphere and backside radiation is minimized. Hence, a high front-to-back ratio (FTBR) can be obtained, resulting in a much safer and more efficient design, since absorption of radiation by the human body is minimal. In addition, a high suppression of unwanted surface waves can be achieved [18]. By selecting SIW technology for the implementation of the backed cavity, a simple low-profile and cost-effective single substrate design can be realized to allow unobtrusive integration in SFIT systems. To overcome the narrowband nature of such topologies, the bandwidth enhancement technique presented in [15] is applied to obtain a textile UWB SIW cavity-backed slot antenna.

The proposed design, depicted in Fig. 1, is based on a rectangular shaped SIW cavity which is split into two halfcavities (half-cavity A and B) by a non-resonant rectangular slot. By carefully determining the dimensions of the slot and both half parts of the cavity, two hybrid mode combinations are excited at two different, neighboring frequencies. The hybrid mode at the lowest resonance frequency consists of a combination of a weak $\mathrm{TE}_{110}$ and a strong $\mathrm{TE}_{120}$ resonance, resulting in fields in both cavity parts which are out-of-phase. This results in a dominant field situated in half-cavity B. At the higher resonance frequency, a strong $\mathrm{TE}_{110}$ and a weak $\mathrm{TE}_{120}$ mode are combined. Then, the fields in both cavity parts are in phase, but exhibit a large difference in magnitude. The dominant field is now situated in half-cavity A. In both cases, the large electric field across the non-resonant slot will cause it to radiate. By selecting both resonance frequencies close enough to each other, both hybrid modes can be merged and the bandwidth can be increased to obtain an UWB antenna. Power is injected into/extracted from the SIW cavity by means of a $50 \Omega$ grounded coplanar waveguide (GCPW) feed line, located in the feed plane at the center point of the largest SIW cavity wall (Fig. 1.c). For measurement convenience, a SMA connector is soldered at the end of a microstrip line that results from extending the inner conductor of the GCPW feed line.

The antenna was designed and optimized by using the transient solver of CST Microwave studio. During the design 
process, we take care that the return loss characteristic exceeds $10 \mathrm{~dB}$ well below $3.4 \mathrm{GHz}$ and exceeds $13 \mathrm{~dB}$ in the region where both hybrid modes merge, to account for variations in material parameters, inaccuracies of the fabrication process and frequency de-tuning caused by bending, crumpling or proximity of the human body. Furthermore, simulations revealed out-of-band backside radiation at higher frequencies when $g_{c p w}$ was too small and/or when $d_{c p w}$ was chosen too large. The antenna dimensions after an extensive optimization process are given in Table I.

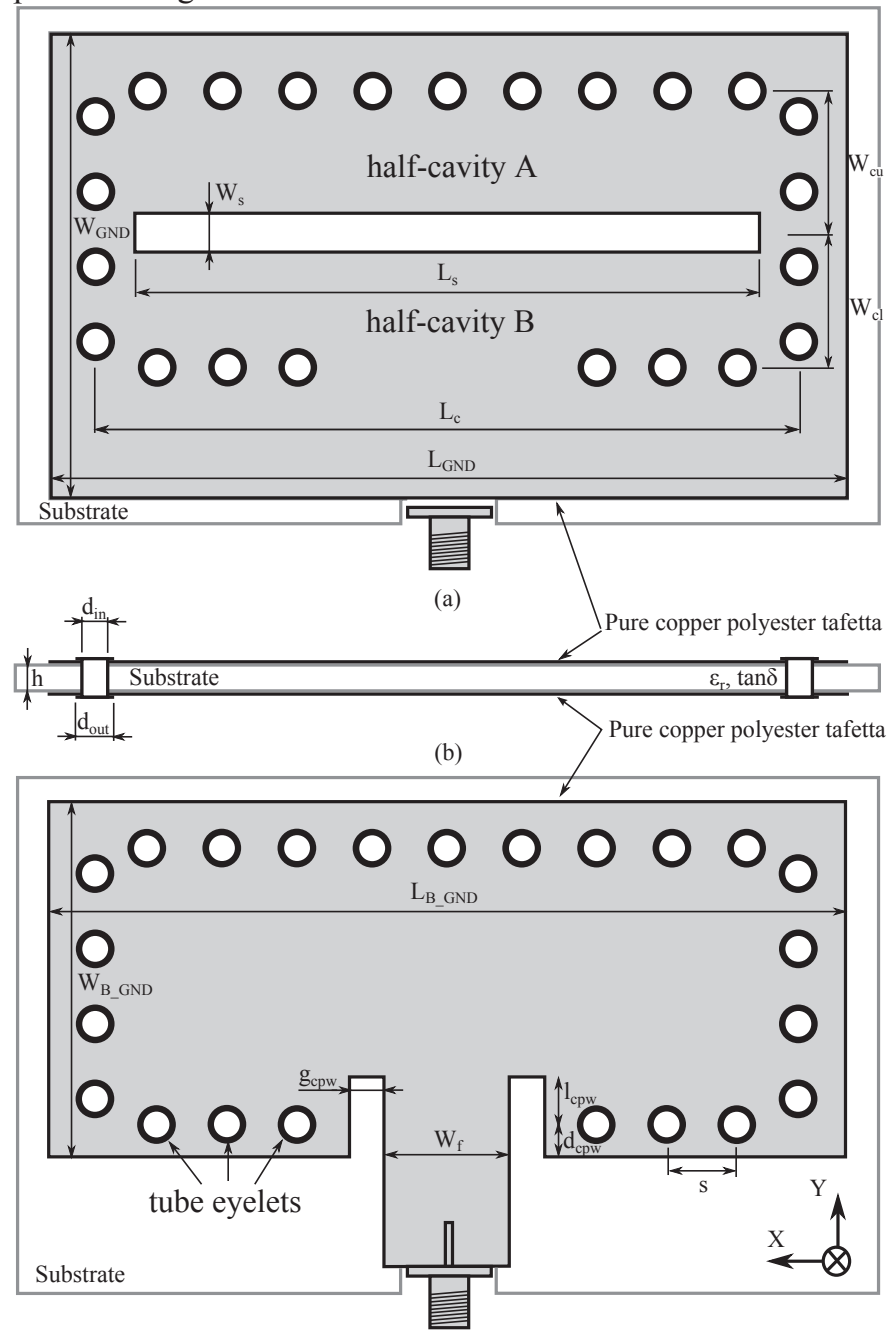

(c)

Fig. 1. (a) Top view: slot plane, (b) cross-sectional view and (c) bottom view: feed plane of the proposed UWB SIW cavity-backed slot

TABLE I

ANTENNA DIMENSIONS

\begin{tabular}{|c|c|c|}
\hline \hline Cavity [mm] & Tube eyelets [mm] & Slot [mm] \\
\hline \hline$L_{c}, W_{c u}, W_{c l}$ & $d_{i n}, d_{o u t}, s$ & $W_{s}, L_{s}$ \\
\hline $75.0,14.34,14.17$ & $4.0,6.0,8.0$ & $4.2,67.0$ \\
\hline \hline \multicolumn{2}{|c|}{ Ground [mm] } & \multicolumn{3}{c|}{ Feed line [mm] } \\
\hline \hline$W_{G N D}, L_{G N D}, W_{B_{G} G N D}, L_{B_{-} G N D}$ & \multicolumn{3}{c|}{$W_{f,} g_{c p w}, l_{c p w}, d_{c p w}$} \\
\hline $49.5,85.0,38.0,85.0$ & \multicolumn{3}{c|}{$13.4,3.7,4.8,3.5$} \\
\hline \hline Substrate $[\mathrm{mm}]$ & $h$ & 3.94 \\
\hline \hline
\end{tabular}

\section{Antenna Materials and Realization}

The materials from which on-body antennas are assembled are of major importance to achieve flexible, light-weight and robust on-body devices. Hence, their selection should be done with the greatest care.

A 3.94-mm-thick closed-cell expanded rubber protective foam, possessing good chemical stability, low moisture regain, and good resistance against oils and solvents, is selected as antenna substrate. It exhibits a relative dielectric permittivity $\varepsilon_{\mathrm{r}}$ of 1.495 and loss tangent $(\tan \delta$ ) of 0.016 at $2.45 \mathrm{GHz}$ [19]. However, a better agreement between measurements and simulations is achieved when a $\tan \delta$ of 0.035 was used in simulations. The slot and feed plane are realized with a copper-coated nylon taffeta electro-textile, with a surface resistivity $R_{S}=0.18 \Omega / \mathrm{sq}$ [19]. After judicious alignment, both conductive layers are glued to the antenna substrate by means of thermally-activated adhesive sheets. Finally, flat flange copper tube eyelets are applied to form the antenna cavity (Fig. 1). Fig. 2 depicts a prototype of our UWB SIW textile antenna.

\section{ANTENNA PERFORMANCE}

First, the return loss characteristic of the antenna in free space conditions was verified in an anechoic room by means of Agilent's N5242A PNA-X Network Analyzer. The simulated and measured reflection coefficients $\left|S_{11}\right|$, depicted in Fig. 3, are in very good agreement. Simulations and measurements demonstrate good impedance matching to $Z_{0}=50 \Omega$ from 3.33 $\mathrm{GHz}$ to $4.66 \mathrm{GHz}$, corresponding with a $-10 \mathrm{~dB}$ impedance bandwidth of $1.33 \mathrm{GHz}$ and a fractional bandwidth of $33 \%$. Hence, the antenna exhibits UWB behavior. Over the entire [3.4-4.8] GHz band, the return loss remains larger than $6 \mathrm{~dB}$.

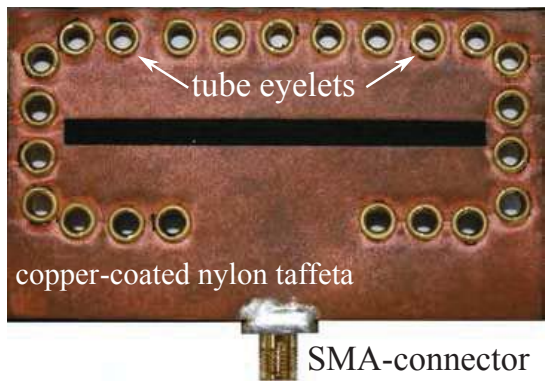

(a)

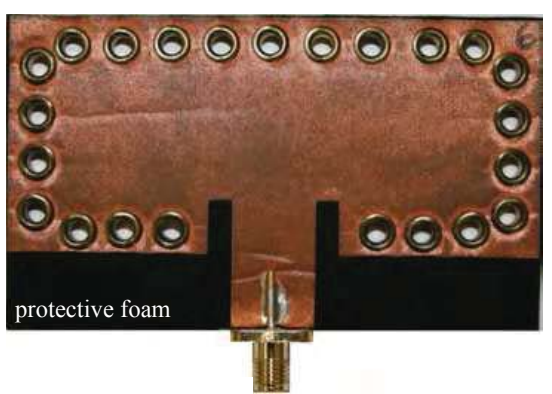

(b)

Fig. 2. Photograph of the slot plane, top view (a), and the feed plane, bottom view (b), of the UWB SIW textile antenna prototype. 


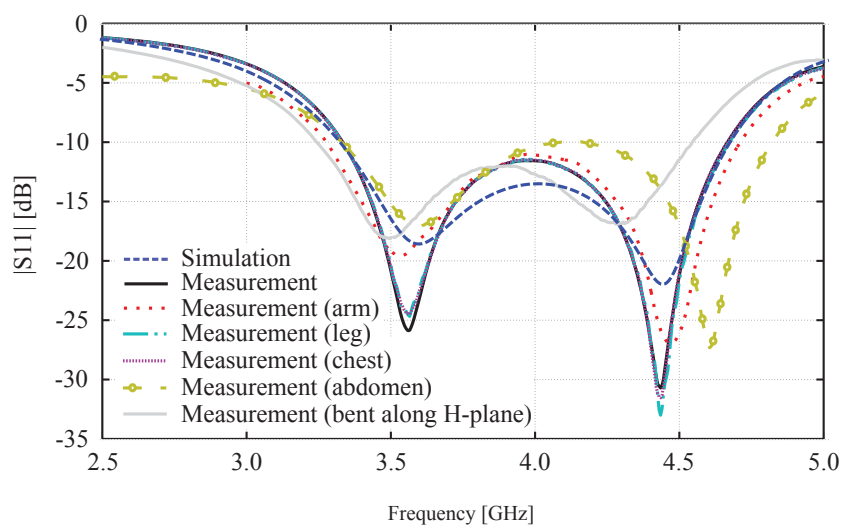

Fig. 3. $\left|S_{I I}\right|$ with respect to $50 \Omega$ when the antenna is deployed under different conditions

The same measurement setup, extended by an orbit/FR positioning system, was used to measure the free-space radiation patterns. Fig. 4 depicts the measured and simulated radiation patterns in the E- and $\mathrm{H}$-plane of the antenna at 3.4 $\mathrm{GHz}, 3.85 \mathrm{GHz}$, and $4.3 \mathrm{GHz}$. Simulations predicted very similar radiation patterns over the entire bandwidth, which is advantageous for minimizing direction-specific distortion of UWB wave forms, and is proven by the measurements in Fig. 4. Simulated and measured antenna gain along broadside, FTBR, and 3dB-beamwidth in the E- and H-plane at the three earlier mentioned frequencies are described in Table II. The measured gain along broadside is slightly higher than the simulated gain at 3.4 and $3.85 \mathrm{GHz}$, whereas the gain at 4.3 $\mathrm{GHz}$ is lower than simulated. A slightly larger backside radiation could be observed at all three frequencies. The measured 3dB-beamwidth along the H-plane is similar at each frequency. A narrower beam is observed compared to simulations, whereas the measured $3 \mathrm{~dB}$-beamwidth along the E-plane slightly increases with frequency. A broader beam is obtained compared to simulations. Generally speaking, we conclude that simulations and measurements agree very well.

Since textile on-body antennas will be worn in proximity of the body, their performance should also be extensively tested when deployed onto the human body and when bent, to be certain that they still perform in more realistic situations. Therefore, on-body $\left|S_{11}\right|$ measurements were performed and depicted in Fig. 3. The antenna was positioned on the arm, leg, chest and abdomen of an average height and weight male test person wearing a T-shirt and jeans. In case of deployment on the leg, the jeans served as spacer. In the other cases, the Tshirt was used as spacer. The antenna was positioned in such a way that bending is prevented as much as possible. The $\left|S_{11}\right|$ remains unchanged when deploying the antenna on the chest or leg, whereas a minor change can be noticed when the antenna is deployed on the arm. The curve corresponding to the measurement on the abdomen exhibits an increase in bandwidth (1.49 GHz) because of a shift in the highest resonance frequency.

Finally, the antenna was bent with a radius of $5 \mathrm{~cm}$ along H-plane. Fig. 3 shows a decrease in both resonance frequencies and in return loss. However, the bandwidth
TABLE II

MEASURED (SIMULATED) ANTENNA CHARACTERISTICS

\begin{tabular}{ccccc}
\hline \hline $\mathrm{f}$ & Gain & FTBR & \multicolumn{2}{c}{ 3dB-beamwidth $\left[^{\circ}\right]$} \\
{$[\mathrm{GHz}]$} & {$[\mathrm{dBi}]$} & {$[\mathrm{dB}]$} & H-plane & E-plane \\
\hline \hline 3.40 & $6.8(5.9)$ & $10.5(11.6)$ & $46(60)$ & $117(111)$ \\
3.85 & $6.5(6.1)$ & $10.5(12.6)$ & $54(63)$ & $123(108)$ \\
4.3 & $4.6(6.3)$ & $7.1(12.3)$ & $46(60)$ & $133(101)$ \\
\hline \hline
\end{tabular}

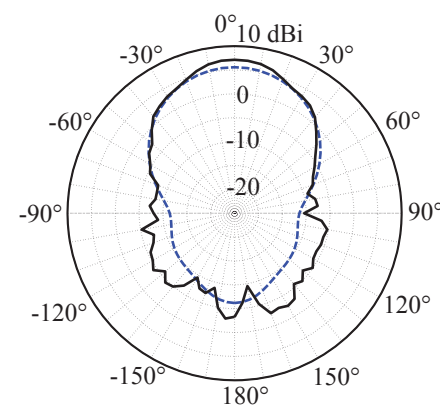

(a)

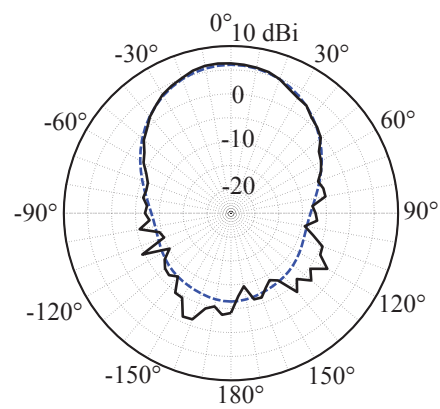

(c)

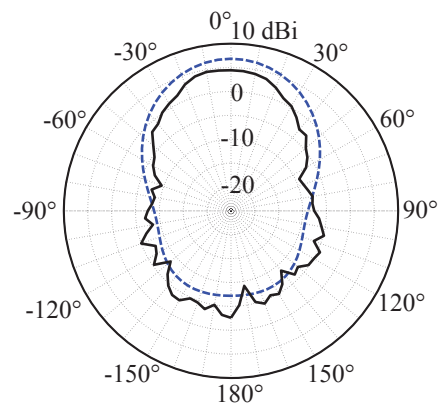

(e)

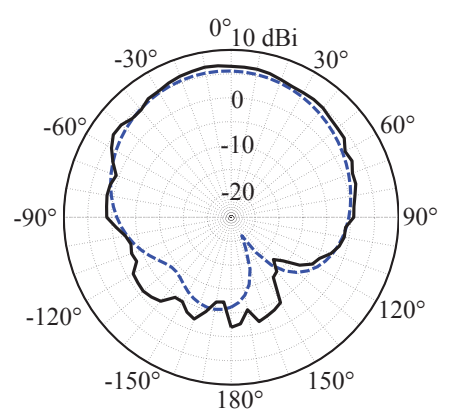

(b)

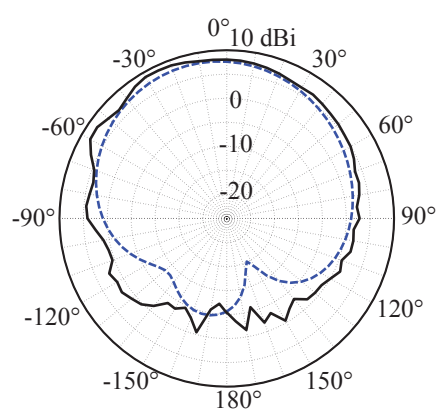

(d)

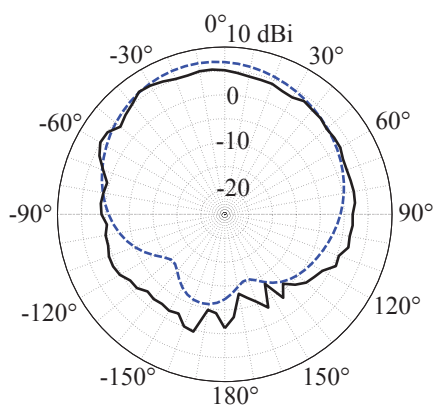

(f)
---- Simulation

- Measurement

Fig. 4. Measured and simulated radiation pattern [dBi] of the antenna at 3.4 $\mathrm{GHz}$ in the H-plane (a) and E-plane (b), at $3.85 \mathrm{GHz}$ in the H-plane (c) and Eplane (d) and at $4.3 \mathrm{GHz}$ in the H-plane (a) and E-plane (b)

reduces only to $1.30 \mathrm{GHz}$, demonstrating its robustness even under extreme bending.

\section{CONCLUSION}

In this contribution, the design of a robust wearable ultrawideband (UWB) textile cavity-backed slot antenna in substrate integrated waveguide (SIW) technology for operation in the low duty cycle restricted [3.4-4.8] GHz band was described. Our design relies on copper tube eyelets and 
textile materials to realize a conformal, light-weight and low profile cavity-backed slot antenna, which exhibits a -10dB impedance bandwidth of $1.33 \mathrm{GHz}$, or $33 \%$, in which a quasiinvariable radiation pattern is maintained. Over the complete [3.4-4.8] GHz band, the return loss exceeds $6 \mathrm{~dB}$. Measurements performed on our prototype after bending and deploying the antenna on different positions of the human body prove its robust performance and very high isolation from the human body. Hence, the potential of exploiting SIW technology for realizing robust UWB wearable antennas is demonstrated.

\section{REFERENCES}

[1] A. Dierck, H. Rogier, and F. Declercq, "A Wearable Active Antenna for Global Positioning System and Satellite Phone," IEEE Trans. Antennas Propag., vol. 61, no. 2, pp. 532-538, Feb. 2013.

[2] M. G. Wing, A. Eklund, and L. D. Kellogg, "Consumer-grade Global Positioning System (GPS) Accuracy and Reliability," J. Forest., vol. 103, no. 4, pp. 169-173, 2005.

[3] P. Meissner, E. Leitinger, M. Fröhle, and K. Witrisal, "Accurate and Robust Indoor Localization Systems using Ultra-wideband Signals," in European Navigation Conference (ENC), Vienna, Austria, Apr. 23-25, 2013.

[4] W. C. Chung, and D. S. Ha, "An Accurate Ultra Wideband (UWB) Ranging for Precision Asset Location," in IEEE Int. Conf. on Ultra Wideband Systems and Technologies, Virginia, USA, Nov. 16-19, 2003, pp. 389-393.

[5] D. Dardari, R. D'Errico, C. Roblin, A. Sibille, and M. Z. Win, "Ultrawide bandwidth RFID: The Next Generation?," Proc. IEEE, vol. 98, no. 9, pp. 1570-1582, Sept. 2010.

[6] Z. Li, W. Dehaene, and G. Gielen, "A 3-tier UWB-based Indoor Localization System for Ultra-Low-Power Sensor Networks," IEEE Trans. Wirel. Commun., vol. 8, no. 6, pp. 2813-2818, 2009.

[7] Federal Communications Commission, "Revision of part 15 of the Commission's Rules Regarding Ultra-Wideband Transmission Systems, First Rep. Order (ET Docket 98-153)," Adopted Feb. 14, 2002. Released Apr. 22, 2002.

[8] Electronic Communications Committee (ECC), "Specific UWB Applications in the bands $3.4-4.8 \mathrm{GHz}$ and $6.8-8.5 \mathrm{GHz}$ Location Tracking Applications for Emergency Services (LAES), Location
Tracking Applications Type 2 (LT2) and Location Tracking and Sensor Applications for Automotive and Transportation Environments (LTA).", Oct. 2011.

[9] W. Q. Malik, C. J. Stevens, and D. J. Edwards, "Ultrawideband Antenna Distortion Compensation," IEEE Trans. Antennas. Propag., vol. 56, no. 7, pp. 1900-1907, 2008.

[10] H. Zhan, J. Ayadi, J. Farserotu, and J.-Y. Le Boudec, "Impulse Radio Ultra-wideband Ranging Based on Maximum Likelihood Estimation," IEEE Trans. Wirel. Commun., vol. 8, no. 12, pp. 5852-5861, Dec. 2009.

[11] D. Dardari, A. Conti, U. Ferner, A. Giorgetti, and M. Z. Win, "Ranging With Ultrawide Bandwidth Signals in Multipath Environments," Proc. IEEE, vol. 97, no. 2, pp. 404-426, Mar. 2009.

[12] T. Kaufmann, and C. Fumeaux, "Wearable Textile Half-Mode Substrate-Integrated Cavity Antenna Using Embroidered Vias," IEEE Antennas and Wireless Propag. Lett. , vol. 12, pp. 805-808, 2013.

[13] R. Moro, S. Agneessens, H. Rogier, and M. Bozzi, "Wearable Textile Antenna in Substrate Integrated Waveguide Technology," IET Electron. Lett., vol. 48, no. 16, pp. 985-987, Aug. 2012.

[14] W. Yang, and J. Zhou, "Wideband Low-Profile Substrate Integrated Waveguide Cavity-Backed E-shaped Patch Antenna," IEEE Antennas Wireless Propag. Lett., vol. 12, pp. 143-146, 2013.

[15] G. Q. Luo, Z. F. Hu, W. J. Li, X. H. Zhang, L. L. Sun, and J. F. Zheng, "Bandwidth-Enhanced Low-Profile Cavity-BackedSlot Antenna by Using Hybrid SIW Cavity Modes," IEEE Trans. Antennas Propag., vol. 60 , no. 4, pp. 1698-1704, 2012.

[16] S. Lemey, F. Declercq, and H. Rogier, "Dual-Band Substrate Integrated Waveguide Textile Antenna With Integrated Solar Harvester," IEEE Antennas Wireless Propag. Lett., vol. 13, no. 1, pp. 269-272, 2014.

[17] A. Dierck, H. Rogier, and F. Declercq, "An Active Wearable Dualband Antenna for GPS and Iridium Satellite Phone Deployed in a Rescue Worker Garment," in IEEE Int. Conf. on RFID- Technologies and Applications, Johor Bahru, Malaysia, Sept 4-5, 2013, pp. 1-5.

[18] G. Q. Luo, Z. F. Hu, L. X. Dong, and L. L. Sun, "Planar slot antenna backed by substrate integrated waveguide cavity," IEEE Antennas Wireless Propag. Lett., vol. 7, no. 1, pp. 236-239, 2008.

[19] F. Declercq, I. Couckuyt, H. Rogier, and T. Dhaene, "Environmental High Frequency Characterization of Fabrics Based on a Novel Surrogate Modelling Antenna Technique," IEEE Trans. Antennas Propag., vol. 61, no. 10, pp. 5200-5213, Oct. 2013. 